\title{
Research on charging strategy of electric vehicle considering user and load curve
}

\author{
Wang Jun ${ }^{1, \mathrm{a}}$, Li Xincong ${ }^{1}$, Xia Minhao ${ }^{1}, \mathrm{Xu} \mathrm{Lin}^{2, \mathrm{~b}^{*}}$ and Wang Bing ${ }^{2}$ \\ ${ }^{1}$ State Grid Shanghai Economic Research Institute, Shanghai, 200120, China \\ ${ }^{2}$ Nanjing Kuanta Information Technology Co., LTD, Nanjing 210006, China
}

\begin{abstract}
With the increasing popularity of electric vehicles, the disordered charging of large-scale electric vehicles will have a great impact on the safe operation of regional distribution network. In order to solve the security problems that may occur in the power grid, this paper uses the time-sharing pricing time division method for EV charging to meet the needs of EV users. Based on this method, a multi-objective optimization model is established, which takes the electric vehicle charging capacity and power as the constraints, and based on the minimum user charging cost and the minimum load curve variance. Then, the model is solved by non-dominated sorting genetic algorithm (NSGA -II), and the optimal compromise solution is extracted by using fuzzy set theory. Finally, the correctness of the proposed model is verified by the example.
\end{abstract}

\section{Introduction}

With the country's strong support for the development of electric vehicles and the increasing awareness of environmental protection, the number of electric vehicles will increase dramatically in the future. However, under the existing technology, charging through the distribution network is the main charging method for EVs. This will increase the power supply pressure of the distribution network and become an important new load for the distribution system. At the same time, with the large-scale development and popularization of electric vehicles, it will inevitably have a major impact on the distribution network, which will cause problems such as overloading of transformers in the distribution network, voltage drops, and increased peak-to-valley differences. Therefore, the impact of large-scale electric vehicle charging ${ }^{1}$ and discharging behavior on the power grid and its charging optimization strategy has become a current research hotspot $^{[1-3]}$.

The optimization of electric vehicle charging strategies mainly involves two objects: EV users and distribution networks.Reference[4] proposed the optimization model and method of the peak-valley electricity price period for the impact of electric vehicle charging and discharging on the peak and valley filling of the power grid, and solved the optimization problem of the peak-valley electricity price period. Reference[5] proposes to use dynamic interpolation to solve the function with the minimum load peak-valley difference as the target, so as to carry out orderly charging control, but the above studies are only from the perspective of the power grid, and do not consider the interests of users. Reference[6] from the perspective of the operator, with the goal of the most profitable charging station, establish a two-stage model to study the optimization strategy of electric vehicle charging. Reference[7] establishes an optimization model aiming at the minimum charging cost of the user and the earliest initial charging time of the battery. Although the user's satisfaction is fully considered, the peak-to-valley load difference is not effectively reduced. Although the above research considers the interests of users or charging stations, it will increase the peak-to-valley difference of the power grid and affect the safe operation of the power grid.

This paper takes the conventional charging methods of electric vehicle charging stations as the research object, comprehensively considers the economics of EV user charging and the safe operation of the power grid, and establishes a multi-objective charging optimization model based on the minimum user charging cost and the minimum variance of the load curve, and adopts The nondominated sorting genetic algorithm (NSGA-II) solves the established model and obtains the Pareto solution set of the multi-objective optimization problem, and then uses the partial fuzzy membership function to solve the Pareto solution set to obtain the optimal compromise solution. Finally, an example is given to verify the effectiveness of the proposed charging strategy.

Corresponding author: ${ }^{\mathrm{a}} 1750906843 @ q q . c o m \quad{ }^{\mathrm{b}} 15850686503 @ 163 . c o m$ 


\section{Charging optimization strategy}

\subsection{Electric vehicle charging time-sharing electricity price period division}

The TOU price of distribution network is set for the nonspecial load in a certain area. After electric vehicles are put into the grid, the local distribution network will be faced with the situation that the division of peak and valley time periods of TOU electricity price is different from the load fluctuation of actual load curve $\mathrm{e}^{[7-8]}$. For example, when the electricity price of the power grid is in the normal period, the load curve of the local distribution network presents a peak state. If $\mathrm{EV}$ users charge under the guidance of this strategy, a large number of EVs may be charged during the peak load period, leading to the phenomenon that the local distribution network appears to exceed the peak. This is not conducive to the safe operation of the power grid, reduces the utilization rate of equipment, and increases the network loss of the local distribution network ${ }^{[12-}$ ${ }^{13]}$. Therefore, on the basis of the original electricity price division period, the ordinary period is further divided, and the TOU electricity price division of electric vehicles is obtained as shown in Table 1:

\begin{tabular}{ccc}
\multicolumn{2}{c}{ Table 1. Electricity price parameter setting } \\
\hline Type & Period & $\begin{array}{c}\text { Charging time-of- } \\
\text { use electricity } \\
\text { price }\end{array}$ \\
\hline $\begin{array}{c}\text { Valley } \\
\text { period }\end{array}$ & $0: 00-8: 00$ & 0.4 \\
$\begin{array}{c}\text { Flat } \\
\text { period }\end{array}$ & $\begin{array}{c}12: 00-14: 30 \\
\text { 21:00-24:00 }\end{array}$ \\
$\begin{array}{c}\text { Peak } \\
\text { period }\end{array}$ & $\begin{array}{c}\text { 8:00-12:00 } \\
14: 30-21: 00\end{array}$ \\
\hline
\end{tabular}

\subsection{Charging optimization model}

Charging optimization model this paper mainly studies the centralized charging station of electric vehicles. When the electric vehicle users access the charging station for charging, the intelligent charging pile of the charging station collects the current remaining battery capacity, type, total capacity and other information from the EV battery management system. The user needs to set the time of vehicle pick-up and the desired state of electric quantity at the end of charging. At the same time, the user can forecast the original load curve of the local distribution network on that day according to the historical load ${ }^{[9]}$. This paper divides one day into 24 time periods with each interval being $1 \mathrm{~h}$, and sets the original load of the local area network as $P_{k}$ in the first time period, and assumes that the battery capacity of electric vehicles is $Q$.

\subsubsection{Objective function}

In order to reduce the adverse impact of the electric vehicle charging load on the overall operation of the power grid, the load variance is used as an objective function, and at the same time considering the economics of EV user charging, the minimum user charging cost is set as another objective function.

(1) Minimum user charge

$$
f_{1}=\min \sum_{t=1}^{T} \sum_{i=1}^{N}\left[c_{f} t_{f}(i)+c_{g} t_{g}(i)+c_{n} t_{n}(i)\right] \Delta t(1)
$$

Where: $\mathrm{N}$ is the number of electric vehicles, $c_{g}$ is the electricity price in the valley period, $c_{n}$ is the electricity price in the flat period, $c_{f}$ is the electricity price in the peak period, $t_{g}, t_{n}, t_{f}$ are the charging time of the vehicle in the valley period, the flat period, and the peak period, $T$ is The number of time periods, $\Delta t$ is the length of the time period, $\Delta t=1 h$.

(2) Minimum load curve variance

The load of the electric vehicle being charged in the $k$ period of time is $P_{e v k}, N$ is the number of electric vehicles, $x_{k}$ is a sign of whether the vehicle is charging, $x_{k}=0$ indicates that the vehicle is not charging, then, $P_{\text {evk }}=\sum_{k=1}^{N} \Delta p_{k} x_{k}$

In the $k$ period, the total load $P_{\text {sumk }}$ of the local distribution network is the sum of the electric vehicle load $P_{e v k}$ and the original load $P_{k}$, then:

$$
P_{\text {sumk }}=P_{\text {evk }}+P_{k}
$$

The variance of the load curve is:

$$
f_{2}=\min \left(\sqrt{\frac{1}{T} \sum_{k=1}^{T}\left[P_{\text {sumk }}-P_{\text {ave }}\right]^{2}}\right)
$$

In the formula: $P_{a v e}$ is the average power of the load curve, $T$ is the time period divided by one day, $T=24$.

\subsubsection{Restrictions}

(1) Target SOC constraints

$$
\left(S O C_{\text {endk }}-S O C_{\text {strk }}\right) B_{k} \leq p_{k}\left(T_{\text {endk }}-T_{\text {strk }}\right)
$$

Where: $S O C_{\text {endk }}$ is the actual level when the $k$-th car leaves, $S O C_{\text {strk }}$ is the level when the $k$-th car starts, $B_{k}$ is the battery capacity of the $k$-th car, $T_{\text {strk }}$ is the charging start time, and $T_{\text {endk }}$ is the charging end time.

(2) Target charge state $S O C$ constraints

$$
S O C_{k} \leq S O C_{\text {endk }} \leq 100
$$

In the formula: $S O C_{k}$ is the expected $S O C$ of the $k$-th car.

(3) Charging power constraints

$0 \leq \Delta p_{k} \leq \Delta p_{k, \text { max }}$ 
In the formula: $\Delta p_{k, \max }$ is the maximum charging power of the electric vehicle.

\section{Model solving method}

\subsection{Model solving method based on NSGA-II algorithm}

Traditional genetic algorithms are mostly used to solve single-objective optimization problems, and the effect of solving multi-objective optimization problems is not ideal. Therefore, traditional algorithms are difficult to solve the problem of electric vehicle charging optimization models with two different optimization goals. Therefore, the NSGA-II algorithm is used to solve it. Compared with the traditional GA algorithm, it introduces a non-dominated quick sorting method, the concept of crowding degree and elite retention strategy. The resulting solution has better convergence and robustness. The calculation steps of the NSGA-II algorithm are as follows:

(1) Initialize the population. 100 populations are randomly generated within the range of fixed decision variables.

(2) Non-dominated sorting and crowding calculation. The newly formed populations are sorted non-dominated to generate different grades, and then the crowding degree is calculated between the different grades.

(3) Selection, crossover and mutation. The selection operator selects individuals based on non-dominated ranking levels and crowded distance. The SBX (binary crossover) operator and polynomial mutation operator perform crossover and mutation operations on the population to form new offspring populations.

(4) Population consolidation. The newly generated child population and the parent population are combined to generate a new population of $2 \mathrm{~N}$.

(5) Non-dominated sorting and crowding calculation. Perform non-dominated sorting and crowding calculations for the new population of $2 \mathrm{~N}$.

(6) The elite retention strategy generates new parent populations. Use the championship selection method to screen a new generation of $\mathrm{N}$ populations.

(7) Repeat steps (2-6) until the genetic algebra meets the requirements.

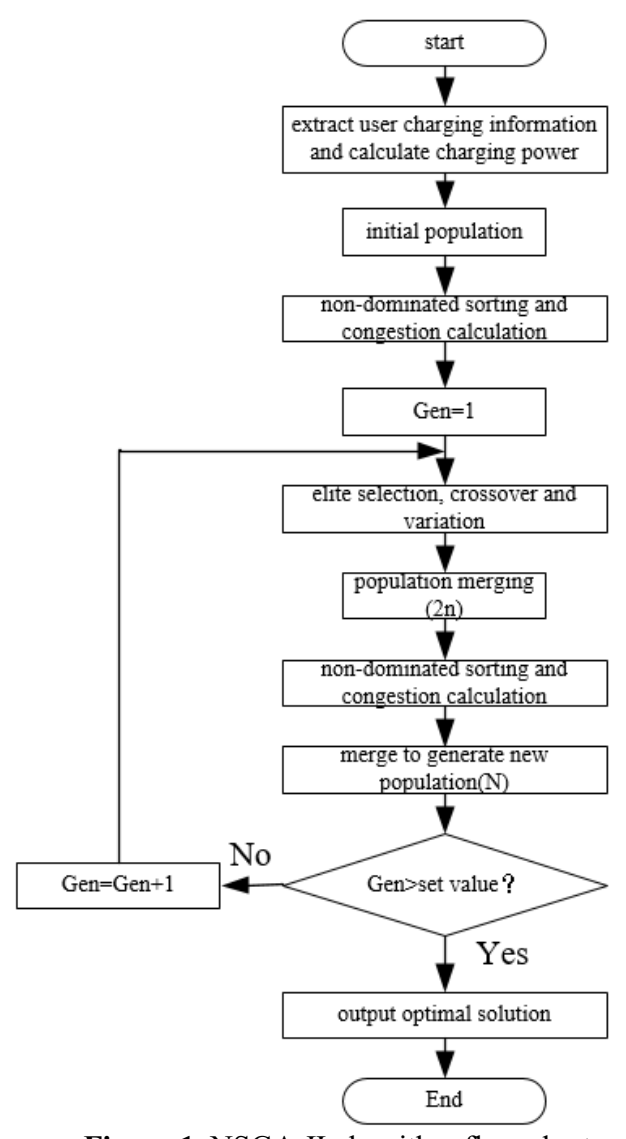

Figure 1. NSGA-II algorithm flow chart

\subsection{Finding the best compromise}

The solution obtained by the NSGA-II algorithm shown in Figure 1 is a set of Pareto solutions, and in actual operation, generally only one optimal solution is selected. Here, we use fuzzy theory to find the optimal compromise solution [11]. When solving the optimal solution of the model, because the optimization goal of the model sought is the minimum problem, a partial fuzzy membership function is selected, and each solution in the solution set is represented by the value of the fuzzy satisfaction function. The fuzzy satisfaction in the set The closer the degree function value is to 1 , the closer the corresponding objective function value is to the optimal solution of the model.

$$
\begin{gathered}
h_{k}=\frac{f_{k}^{\max }-f_{k}}{f_{k}^{\max }-f_{k}^{\min }}, f_{k}^{\min } \leq f_{k} \leq f_{k}^{\max } \\
h=\frac{1}{\omega} \sum_{k=1}^{\omega} h_{k}
\end{gathered}
$$

In the formula: $f_{k}$ is the function value of the optimization goal, $f_{k}^{\max }$ and $f_{k}^{\text {min }}$ are the maximum and minimum values of the optimization goal. $h_{k}$ is the membership function value of objective $k$, and $\omega$ is the number of optimization objective functions. According to formula (8) and formula (9), the $h$ value corresponding to each element in the solution set is obtained, and then the objective function value corresponding to the largest $h$ in the set is selected as the optimal compromise solution. 


\section{Example analysis}

\subsection{Simulation parameter setting}

In order to verify the optimization model and conclusion of this paper, we take all charging stations in a certain region (population of 1 million or so, $2 \%$ of the total population is predicted to be the number of electric vehicles) as an example. The typical daily load curve of this area is shown in Figure 2:

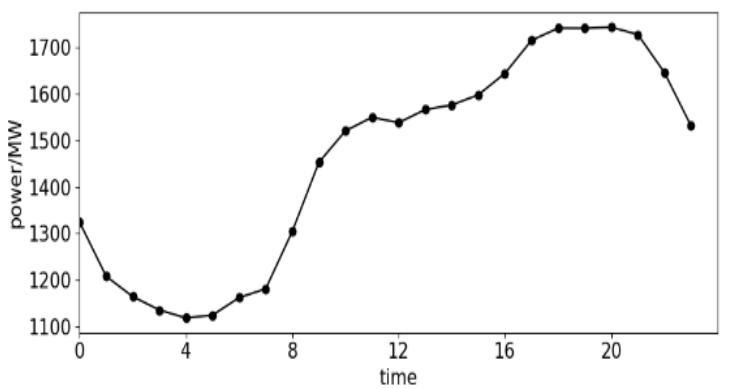

Figure 2 .Typical daily load curve.

The arrival time of electric vehicle approximately obeys the normal distribution of mean 9 variance 0.5 . The initial $S O C$ of the vehicle obeys the uniform distribution between (0.2-0.6). The battery capacity of the electric vehicle is $24 \mathrm{kw}$, and the charging power of the charger is $5 \mathrm{kw}$. Because the guidance degree of the user by the charging strategy is unknown, the response coefficient $\eta$ of the user charging strategy is introduced. $\eta$ is the percentage of the total number of electric private cars that can respond to the charging strategy affected by the charging strategy.

\subsection{Analysis of simulation results}

The population number is 100 , the maximum number of iterations is 500 , the cross rate is 0.8 , and the variation rate is 0.2 . The initial user response coefficient $\eta$ is 0.5 , and then use MATLAB software to solve the multi-objective optimization model, and finally get a group of results.

As shown in Figure 3, the Pareto solution set obtained is relatively smooth, which shows the effectiveness of the algorithm for model solution; with the increase of charging cost, the load standard deviation is decreasing, because when the charging cost is very low, a large number of users charge in the valley price period, resulting in a new load peak, resulting in a large fluctuation of the grid, increasing the load variance.

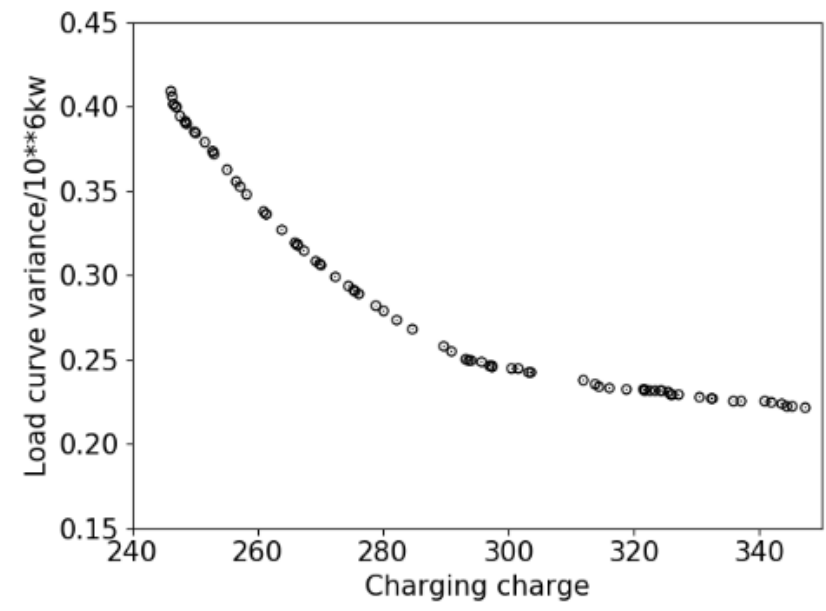

Figure 3. Pareto front

In order to study the effect of charging optimization strategy on different user responsiveness, $25 \%, 50 \%$ and $75 \%$ of user responsiveness are optimized and simulated respectively, and the results are shown in Table 2 . It should be noted that when the user's response degree is 0 , it corresponds to the disordered charging of the electric vehicle, that is, as long as the electric vehicle needs to be charged, it is connected to the charging pile to charge at the maximum power until the desired SOC is achieved.

Table 2. Results under different user response factors

\begin{tabular}{cccc}
$\begin{array}{c}\text { User } \\
\text { response } \\
\text { factor } \boldsymbol{\eta}\end{array}$ & $\begin{array}{c}\text { Number of } \\
\text { controlled } \\
\text { vehicles }\end{array}$ & $\begin{array}{c}\text { Load } \\
\text { curve } \\
\text { variance } \\
\text { /kw }\end{array}$ & $\begin{array}{c}\text { Chargin } \\
\text { g charge }\end{array}$ \\
\hline 0 & 0 & 413854.3 & 449.926 \\
$25 \%$ & 5000 & 374699.1 & 366.621 \\
$50 \%$ & 10000 & 331472.8 & 278.157 \\
$75 \%$ & 15000 & 299781.6 & $57.2 \%$ \\
\hline
\end{tabular}

It can be seen from table 2 that the load variance of the power system is the largest when charging out of order, which leads to the decrease of the utilization rate of power resources, and will have a negative impact on the power grid. At the same time, the charging cost of users is the highest. The higher the response coefficient of the user's charging strategy is, the higher the vehicle's response to the charging strategy is, the smaller the variance of the total load is, reducing by $9.5 \%, 18.9 \%$ and $26.5 \%$ respectively, which shows that the charging strategy used in this model can smooth the load curve to a certain extent and reduce the variance rate of the load curve. At the same time, the user's charge cost decreases with the increase of the response degree of the charging strategy, which is $18.5 \%, 38.2 \%$ and $57.2 \%$ lower than the disordered charging. It can be seen that the charging optimization strategy can significantly reduce the user's charge cost and improve the user's satisfaction. In conclusion, with more and more vehicles responding to the charging strategy, the overall load variance of the system will gradually reduce, and the user charging cost will also reduce, which shows that the established optimization model can not only make the system run more safely, but also improve the user satisfaction. 


\section{Conclusion}

Aiming at the problem of electric vehicle charging optimization, this paper presents a multi-objective charging optimization model, which considers the user's expected SOC and charging power constraints, and takes the minimum user's charging cost and the minimum load curve variance as the optimization objective. The simulation results obtained by studying the response of different charging strategies verify the feasibility and accuracy of the model, it is proved that the strategy can effectively reduce the charging cost and load variance, and enhance the economy and stability of the system operation. This paper assumes that all electric vehicles are of one type, and later research can be carried out for different types of electric vehicles.

\section{References}

1. GAO Ciwei, ZHANG Liang, A Survey of Influence of Electrics Vehicle Charging on Power Grid, Power System Technology. 02, 133-137(2011)

2. MA Lingling, YANG Jun, Review on impact of electric car charging and discharging on power grid, Power System Protection And Control.41, 140148(2014)

3. SORTOMME E,HINDIM M, MACPHERSON J. Coordinated charging of plug-in hybrid electric vehicles to minimize distribution system losses. IEEE Trans on Smart Grid. 2(1), 198-205(2011)

4. GE Shaoyun, WANG Long, LIU Hong, An Optimization Model of Peak-Valley Price TimeInterval Considering Vehicle-to-Grid, Power System Technology. 37,(8), 2316-2321(2013)

5. QIU Yuehao, Intelligent strategy on coordinated charging of PHEV with TOU price, Asia-Pacific Power and Energy Engineering Conference, APPEEC 2011-Proceedings.(2011)

6. ZHANG Liang, YAN Zheng, FENG Donghan, TwoStage Optimization Model Based Coordinated Charging for EV Charging Station, Power System Technology. 38, 967-973(2014)

7. SUN Xiaoming, WANG Wei, SU Su, Coordinated Charging Strategy for Electric Vehicles Based on Time-of-use Price, Automation of Electric Power. 37, 191-195 (2017)

8. ZHANG Cong, XU Xiaohui, SUN Haishun, Smart charging strategy of large-scale electric vehicles based on adaptive genetic algorithm, Power System Protection And Control. 14, 29-34(2014)

9. HOU Chaojian, HU Qunfeng, TAN Fuzhong, Multiobjective optimization model of collaborative WP-EV dispatch considering demand response, Electric Power Automation Equipment. 36, 22-27(2016)

10. TONG Jingjing, WEN Junqiang, WANG Dan,Statistical Model for Charging Power Demand of Electric Vehicles, Power System Technology. 31, 126-130(2011)
11. TIAN Liting, SHI Shuanglong, JIA Zhuo, Multiobjective optimization charging strategy for plug-in electric vehicles based on time-of-use prices, Power System Protection and Control. 44, 17-23(2016)

12. YAO Weifeng, ZHAO Junhua, WEN Fushuan, $A$ Charging and Discharging Dispatching Strategy for Electric Vehicles Based on Bi-level Optimization, Automation of Electric Power. 36, 30-37(2012)

13. CHANG Fangyu, HUANG Mei, ZHANG Weige, Research on Coordinated Charging of Electric Vehicles Based on TOU Charging Price, Power System Technology. 19, 2609-2615(2016) 